\title{
Predicting the Leptonic Dirac CP Violation Phase from Sum Rules
}

\author{
Ivan Girardi \\ SISSA/INFN, Trieste, Italy \\ E-mail: igirardiesissa.it

\section{S. T. Petcov*} \\ SISSA/INFN, Trieste, Italy and Kavli IPMU, Kashiwa, Japan \\ E-mail: petcovesissa.it
}

\author{
Arsenii Titov ${ }^{\dagger}$ \\ SISSA/INFN, Trieste, Italy \\ E-mail: atitovesissa.it
}

\begin{abstract}
Establishing the status of the CP symmetry in the lepton sector is one of the major goals of the programme of future research in neutrino physics. In the reference 3-neutrino mixing scheme CP-violating effects in neutrino oscillations can be caused by the Dirac CP violation phase $\delta$ present in the $3 \times 3$ unitary neutrino mixing matrix $U$. Using the fact that $U=U_{e}^{\dagger} U_{v}$, where $U_{e}$ and $U_{v}$ are $3 \times 3$ unitary matrices which diagonalise respectively the charged lepton and neutrino mass matrices, we consider in a systematic way forms of $U_{e}$ and $U_{v}$ allowing us to express $\delta$ as a function of the neutrino mixing angles present in $U$ and the angles contained in $U_{v}$. After obtaining sum rules for $\cos \delta$, we consider several forms of $U_{v}$ dictated by, or associated with, symmetries, such as tri-bimaximal, bimaximal, etc., for which the angles in $U_{v}$ are fixed. For each of these forms and forms of $U_{e}$ allowing to reproduce the measured values of the neutrino mixing angles, we construct the likelihood function for $\cos \delta$, using the prospective uncertainties in the determination of the neutrino mixing angles. Our results show that the measurement of $\delta$ along with improvement of the precision on the neutrino mixing angles can provide unique information as regards the possible existence of symmetry in the lepton sector.
\end{abstract}

The European Physical Society Conference on High Energy Physics

22-29 July 2015

Vienna, Austria

*Also at: Institute of Nuclear Research and Nuclear Energy, Bulgarian Academy of Sciences, Sofia, Bulgaria.

† Speaker. 


\section{Introduction}

Understanding the origin of the observed pattern of neutrino mixing and establishing the status of the CP symmetry in the lepton sector are among the highest priority goals of the programme of future research in neutrino physics (see, e.g., [1]). One of the major experimental efforts within this programme will be dedicated to the searches for CP-violating effects in neutrino oscillations (see, e.g., $[2,3])$. In the reference 3-neutrino mixing scheme with three light massive neutrinos we are going to consider (see, e.g., [1]), the CP-violating effects in neutrino oscillations can be caused, as is well known, by the Dirac CP violation (CPV) phase present in the Pontecorvo, Maki, Nakagawa, Sakata (PMNS) neutrino mixing matrix. Predictions for the Dirac CPV phase in the lepton sector can be, and were, obtained, in particular, combining the phenomenological approach, developed in $[4,5,6,7,8]$, with symmetry considerations. In this approach one exploits the fact that the PMNS mixing matrix $U$ has the form [6]:

$$
U=U_{e}^{\dagger} U_{v}=\tilde{U}_{e}^{\dagger} \Psi \tilde{U}_{v} Q_{0}
$$

where $U_{e}$ and $U_{v}$ are $3 \times 3$ unitary matrices originating from the diagonalisation respectively of the charged lepton and neutrino mass matrices. In Eq. (1.1) $\tilde{U}_{e}$ and $\tilde{U}_{v}$ are CKM-like $3 \times 3$ unitary matrices, and $\Psi$ and $Q_{0}$ are diagonal phase matrices each containing in the general case two CPV phases:

$$
\Psi=\operatorname{diag}\left(1, e^{-i \psi}, e^{-i \omega}\right), \quad Q_{0}=\operatorname{diag}\left(1, e^{i \frac{\xi_{21}}{2}}, e^{i \frac{\xi_{31}}{2}}\right)
$$

The phases in the matrix $Q_{0}$ contribute to the Majorana phases in the PMNS matrix [9]. It is further assumed that, up to subleading perturbative corrections (and phase matrices), the PMNS matrix $U$ has a specific known form $\tilde{U}_{v}$ that is dictated by continuous and/or discrete symmetries, or by arguments related to symmetries. This assumption seems very natural in view of the observation that the measured values of the three neutrino mixing angles differ from certain possible symmetry values by subdominant corrections. Indeed, in terms of angles, the best fit values of the three neutrino mixing parameters $\sin ^{2} \theta_{12}, \sin ^{2} \theta_{23}$ and $\sin ^{2} \theta_{13}$, obtained in the global analysis of neutrino oscillation data performed in [10], ${ }^{1}$ imply: $\theta_{12} \approx \pi / 5.34, \theta_{13} \approx \pi / 20$ and $\theta_{23} \approx \pi / 4.35$. Thus, $\theta_{12}$ deviates from the possible symmetry value $\pi / 4$ by approximately $0.2, \theta_{13}$ deviates from 0 (or from $\pi / 10$ ) by approximately 0.16 , and $\theta_{23}$ deviates from the symmetry value $\pi / 4$ by approximately 0.06 , where we used $\sin ^{2} \theta_{23}=0.437$.

Widely discussed symmetry forms of $\tilde{U}_{v}$ include: i) tri-bimaximal (TBM) form [5, 12], ii) bimaximal (BM) form, or due to a symmetry corresponding to the conservation of the lepton charge $L^{\prime}=L_{e}-L_{\mu}-L_{\tau}$ (LC) [13, 14], iii) golden ratio type A (GRA) form [15], iv) golden ratio type B (GRB) form [16], and v) hexagonal (HG) form [17]. For all these forms the matrix $\tilde{U}_{v}$ represents a product of two orthogonal matrices describing rotations in the 1-2 and 2-3 planes on fixed angles $\theta_{12}^{v}$ and $\theta_{23}^{v}$ :

$$
\tilde{U}_{v}=R_{23}\left(\theta_{23}^{v}\right) R_{12}\left(\theta_{12}^{v}\right),
$$

\footnotetext{
${ }^{1}$ Similar results were obtained in the global analysis of neutrino oscillation data performed in [11].
} 
where

$$
R_{12}\left(\theta_{12}^{v}\right)=\left(\begin{array}{ccc}
\cos \theta_{12}^{v} & \sin \theta_{12}^{v} & 0 \\
-\sin \theta_{12}^{v} & \cos \theta_{12}^{v} & 0 \\
0 & 0 & 1
\end{array}\right), \quad R_{23}\left(\theta_{23}^{v}\right)=\left(\begin{array}{ccc}
1 & 0 & 0 \\
0 & \cos \theta_{23}^{v} & \sin \theta_{23}^{v} \\
0-\sin \theta_{23}^{v} & \cos \theta_{23}^{v}
\end{array}\right) .
$$

For all the symmetry forms quoted above one has $\theta_{23}^{v}=-\pi / 4$. The forms differ by the value of the angle $\theta_{12}^{v}$, and, correspondingly, of $\sin ^{2} \theta_{12}^{v}$ : for the TBM, BM (LC), GRA, GRB and HG forms we have, respectively, $\sin ^{2} \theta_{12}^{v}=1 / 3,1 / 2,(2+r)^{-1} \approx 0.276,(3-r) / 4 \approx 0.345$, and $1 / 4, r$ being the golden ratio, $r=(1+\sqrt{5}) / 2$.

As is clear from the preceding discussion, the values of the angles in the matrix $\tilde{U}_{v}$, which are fixed by symmetry arguments, typically differ from the values determined experimentally by relatively small perturbative corrections. In the approach we are following, the requisite corrections to the symmetry values are provided by the angles in the matrix $\tilde{U}_{e}$. The matrix $\tilde{U}_{e}$ in the general case depends on three angles and one phase [6]. For certain forms of $\tilde{U}_{e}$ and $\tilde{U}_{v}$ the Dirac phase $\delta$ present in the PMNS mixing matrix satisfies sum rules by which it is expressed in terms of the neutrino mixing angles measured in the neutrino oscillation experiments and the angles in the matrix $\tilde{U}_{v}$ whose values are fixed (see, e.g., $\left.[18,19,20]\right)$.

In the present note we recapitulate the sum rules for $\cos \delta$ derived in $[19,20]$ for the following forms of $\tilde{U}_{v}$ and $\tilde{U}_{e}:^{2}$
A. $\tilde{U}_{v}=R_{23}\left(\theta_{23}^{v}\right) R_{12}\left(\theta_{12}^{v}\right)$ and
(i) $\tilde{U}_{e}=R_{12}^{-1}\left(\theta_{12}^{e}\right)$,
(ii) $\tilde{U}_{e}=R_{13}^{-1}\left(\theta_{13}^{e}\right)$,
(iii) $\tilde{U}_{e}=R_{23}^{-1}\left(\theta_{23}^{e}\right) R_{12}^{-1}\left(\theta_{12}^{e}\right)$, and (iv) $\tilde{U}_{e}=R_{23}^{-1}\left(\theta_{23}^{e}\right) R_{13}^{-1}\left(\theta_{13}^{e}\right)$;
B. $\tilde{U}_{v}=R_{23}\left(\theta_{23}^{v}\right) R_{13}\left(\theta_{13}^{v}\right) R_{12}\left(\theta_{12}^{v}\right)$ and
(v) $\tilde{U}_{e}=R_{12}^{-1}\left(\theta_{12}^{e}\right)$, and
(vi) $\tilde{U}_{e}=R_{13}^{-1}\left(\theta_{13}^{e}\right)$.

We would like to notice here that in the case of $\tilde{U}_{e}=R_{23}^{-1}\left(\theta_{23}^{e}\right)$ the parameter $\sin ^{2} \theta_{13}=\sin ^{2} \theta_{13}^{v}$, i.e., the reactor angle does not get corrected and, in particular, remains zero for the form of $\tilde{U}_{v}$ given in point A. This is why we do not consider this case. After presenting the sum rules of interest, we derive predictions for $\cos \delta$, performing a statistical analysis using the prospective uncertainties in the determination of the neutrino mixing parameters $\sin ^{2} \theta_{12}, \sin ^{2} \theta_{13}$ and $\sin ^{2} \theta_{23}$.

\section{Sum Rules}

We start with the first case, in which $\tilde{U}_{e}=R_{12}^{-1}\left(\theta_{12}^{e}\right)$ and $\tilde{U}_{v}=R_{23}\left(\theta_{23}^{v}\right) R_{12}\left(\theta_{12}^{v}\right)$. According to Eq. (1.1), the PMNS matrix $U$ reads

$$
U=R_{12}\left(\theta_{12}^{e}\right) \Psi R_{23}\left(\theta_{23}^{v}\right) R_{12}\left(\theta_{12}^{v}\right) Q_{0} .
$$

We consider the case of arbitrary fixed values of the angles $\theta_{12}^{v}$ and $\theta_{23}^{v}$. Using Eq. (2.1) and the standard parametrisation of the PMNS mixing matrix (see, e.g., [1]), one finds ${ }^{3}$

$$
\sin ^{2} \theta_{23}=\frac{\left|U_{\mu 3}\right|^{2}}{1-\left|U_{e 3}\right|^{2}}=\frac{\sin ^{2} \theta_{23}^{v}-\sin ^{2} \theta_{13}}{1-\sin ^{2} \theta_{13}}
$$

\footnotetext{
${ }^{2}$ We would like to point out that all the cases considered in this study can be realised when a discrete (lepton) flavour symmetry group $G_{f}$ is fully broken in the charged lepton sector and is broken to a residual symmetry $G_{v}$ in the neutrino sector which fixes the matrix $\tilde{U}_{v}$ (see, e.g., [21]).

${ }^{3}$ In the present note we give only results. The details of the relevant derivations can be found in [20].
} 
and

$$
\begin{aligned}
\cos \delta= & \frac{\left(\cos 2 \theta_{13}-\cos 2 \theta_{23}^{v}\right)^{\frac{1}{2}}}{\sqrt{2} \sin 2 \theta_{12} \sin \theta_{13}\left|\cos \theta_{23}^{v}\right|}\left[\cos 2 \theta_{12}^{v}+\left(\sin ^{2} \theta_{12}\right.\right. \\
& \left.\left.-\cos ^{2} \theta_{12}^{v}\right) \frac{2 \sin ^{2} \theta_{23}^{v}-\left(3+\cos 2 \theta_{23}^{v}\right) \sin ^{2} \theta_{13}}{\cos 2 \theta_{13}-\cos 2 \theta_{23}^{v}}\right] .
\end{aligned}
$$

As can be seen from Eq. (2.2), in the scheme under consideration $\sin ^{2} \theta_{23}$ can deviate from $\sin ^{2} \theta_{23}^{v}$ only by approximately the quantity $\cos ^{2} \theta_{23}^{v} \sin ^{2} \theta_{13}$, namely, $\sin ^{2} \theta_{23} \approx \sin ^{2} \theta_{23}^{v}-\cos ^{2} \theta_{23}^{v} \sin ^{2} \theta_{13}$, where we have used the fact that $\sin ^{2} \theta_{13} \ll 1$. In particular, for all the symmetry forms described in the Introduction, $\theta_{23}^{v}=-\pi / 4$, and $\sin ^{2} \theta_{23} \approx 0.5-0.5 \sin ^{2} \theta_{13}$, which, taking into account the best fit value of $\sin ^{2} \theta_{13}=0.0234$, implies that $\theta_{23} \approx \pi / 4$.

In the second case, $\tilde{U}_{e}=R_{13}^{-1}\left(\theta_{13}^{e}\right), \tilde{U}_{v}=R_{23}\left(\theta_{23}^{v}\right) R_{12}\left(\theta_{12}^{v}\right)$, and we have for $U$ :

$$
U=R_{13}\left(\theta_{13}^{e}\right) \Psi R_{23}\left(\theta_{23}^{v}\right) R_{12}\left(\theta_{12}^{v}\right) Q_{0} .
$$

This parametrisation of the PMNS matrix leads to

$$
\sin ^{2} \theta_{23}=\frac{\left|U_{\mu 3}\right|^{2}}{1-\left|U_{e 3}\right|^{2}}=\frac{\sin ^{2} \theta_{23}^{v}}{1-\sin ^{2} \theta_{13}}
$$

and

$$
\begin{aligned}
\cos \delta= & -\frac{\left(\cos 2 \theta_{13}+\cos 2 \theta_{23}^{v}\right)^{\frac{1}{2}}}{\sqrt{2} \sin 2 \theta_{12} \sin \theta_{13}\left|\sin \theta_{23}^{v}\right|}\left[\cos 2 \theta_{12}^{v}+\left(\sin ^{2} \theta_{12}\right.\right. \\
& \left.\left.-\cos ^{2} \theta_{12}^{v}\right) \frac{2 \cos ^{2} \theta_{23}^{v}-\left(3-\cos 2 \theta_{23}^{v}\right) \sin ^{2} \theta_{13}}{\cos 2 \theta_{13}+\cos 2 \theta_{23}^{v}}\right] .
\end{aligned}
$$

As in the first case, the value of $\sin ^{2} \theta_{23}$ is fixed by the values of $\theta_{23}^{v}$ and $\theta_{13}$. Expanding in $\sin ^{2} \theta_{13}$, we obtain $\sin ^{2} \theta_{23} \approx \sin ^{2} \theta_{23}^{v}+\sin ^{2} \theta_{23}^{v} \sin ^{2} \theta_{13}$, and for $\theta_{23}^{v}=-\pi / 4, \sin ^{2} \theta_{23} \approx 0.5+0.5 \sin ^{2} \theta_{13}$, implying again almost maximal atmospheric mixing. Thus, as we can see, in the schemes with two rotations from the neutrino sector with $\theta_{23}^{v}=-\pi / 4$ and only one rotation from the charged lepton sector, it is not possible, in particular, to reproduce the current best fit value of $\sin ^{2} \theta_{23}$. However, at present this value is determined with a relatively large uncertainty [10,11]. Future experiments will establish whether $\sin ^{2} \theta_{23}$ deviates significantly from 0.5 or not. If it deviates, one can get the requisite correction introducing a second rotation matrix in $\tilde{U}_{e}$ arising from the charged lepton sector.

In the third case, when $\tilde{U}_{e}=R_{23}^{-1}\left(\theta_{23}^{e}\right) R_{12}^{-1}\left(\theta_{12}^{e}\right)$ and $\tilde{U}_{v}=R_{23}\left(\theta_{23}^{v}\right) R_{12}\left(\theta_{12}^{v}\right)$, the PMNS matrix takes the following form:

$$
U=R_{12}\left(\theta_{12}^{e}\right) R_{23}\left(\theta_{23}^{e}\right) \Psi R_{23}\left(\theta_{23}^{v}\right) R_{12}\left(\theta_{12}^{v}\right) Q_{0}
$$

This form of $U$ leads to the sum rule for $\cos \delta$, which was first derived for an arbitrary fixed value of $\theta_{12}^{v}$ in [19]. This sum rule reads

$$
\cos \delta=\frac{\tan \theta_{23}}{\sin 2 \theta_{12} \sin \theta_{13}}\left[\cos 2 \theta_{12}^{v}+\left(\sin ^{2} \theta_{12}-\cos ^{2} \theta_{12}^{v}\right)\left(1-\cot ^{2} \theta_{23} \sin ^{2} \theta_{13}\right)\right] .
$$


For the specific values of $\theta_{12}^{v}=\pi / 4$ and $\theta_{12}^{v}=\sin ^{-1}(1 / \sqrt{3})$, i.e., for the BM (LC) and TBM forms of $\tilde{U}_{v}$, Eq. (2.8) reduces to the expressions for $\cos \delta$ derived first in [18]. Although Eq. (2.8) was derived in [19] for $\theta_{23}^{v}=-\pi / 4$, it holds for an arbitrary fixed value of $\theta_{23}^{v}$ [20].

A similar sum rule is found in the fourth case, in which $\tilde{U}_{e}=R_{23}^{-1}\left(\theta_{23}^{e}\right) R_{13}^{-1}\left(\theta_{13}^{e}\right)$ and $\tilde{U}_{v}=$ $R_{23}\left(\theta_{23}^{v}\right) R_{12}\left(\theta_{12}^{v}\right)$ result in

$$
U=R_{13}\left(\theta_{13}^{e}\right) R_{23}\left(\theta_{23}^{e}\right) \Psi R_{23}\left(\theta_{23}^{v}\right) R_{12}\left(\theta_{12}^{v}\right) Q_{0} .
$$

Comparing this parametrisation with the standard parametrisation of the PMNS matrix, one finds

$$
\cos \delta=-\frac{\cot \theta_{23}}{\sin 2 \theta_{12} \sin \theta_{13}}\left[\cos 2 \theta_{12}^{v}+\left(\sin ^{2} \theta_{12}-\cos ^{2} \theta_{12}^{v}\right)\left(1-\tan ^{2} \theta_{23} \sin ^{2} \theta_{13}\right)\right] .
$$

As in the previous case, the sum rule in Eq. (2.10) is valid for any fixed value of $\theta_{23}^{v}$.

Finally, we consider the cases with a third rotation matrix in $\tilde{U}_{v}$ arising from the neutrino sector. Non-zero values of $\theta_{13}^{v}$ are inspired by certain types of flavour symmetries (see, e.g., [22]). yields

In the fifth case, $\tilde{U}_{e}=R_{12}^{-1}\left(\theta_{12}^{e}\right), \tilde{U}_{v}=R_{23}\left(\theta_{23}^{v}\right) R_{13}\left(\theta_{13}^{v}\right) R_{12}\left(\theta_{12}^{v}\right)$, and the PMNS matrix $U$

$$
U=R_{12}\left(\theta_{12}^{e}\right) \Psi R_{23}\left(\theta_{23}^{v}\right) R_{13}\left(\theta_{13}^{v}\right) R_{12}\left(\theta_{12}^{v}\right) Q_{0}
$$

In this set-up the value of $\sin ^{2} \theta_{23}$ is fixed by the values of $\theta_{23}^{v}, \theta_{13}^{v}$ and $\theta_{13}$ :

$$
\sin ^{2} \theta_{23}=\frac{\left|U_{\mu 3}\right|^{2}}{1-\left|U_{e 3}\right|^{2}}=1-\frac{\cos ^{2} \theta_{23}^{v} \cos ^{2} \theta_{13}^{v}}{1-\sin ^{2} \theta_{13}} .
$$

The sum rule of interest in this case reads

$$
\begin{aligned}
\cos \delta= & \frac{1}{\sin 2 \theta_{12} \sin \theta_{13}\left|\cos \theta_{13}^{v} \cos \theta_{23}^{v}\right|\left(\cos ^{2} \theta_{13}-\cos ^{2} \theta_{13}^{v} \cos ^{2} \theta_{23}^{v}\right)^{\frac{1}{2}}} \\
& \times\left[\left(\cos ^{2} \theta_{13}-\cos ^{2} \theta_{13}^{v} \cos ^{2} \theta_{23}^{v}\right) \sin ^{2} \theta_{12}+\cos ^{2} \theta_{12} \sin ^{2} \theta_{13} \cos ^{2} \theta_{13}^{v} \cos ^{2} \theta_{23}^{v}\right. \\
& \left.-\cos ^{2} \theta_{13}\left(\cos \theta_{12}^{v} \sin \theta_{13}^{v} \cos \theta_{23}^{v}-\sin \theta_{12}^{v} \sin \theta_{23}^{v}\right)^{2}\right] .
\end{aligned}
$$

Thus, $\cos \delta$ depends on $\theta_{12}, \theta_{13}$ and all the three angles in the matrix $\tilde{U}_{v}$, i.e., $\theta_{12}^{v}, \theta_{23}^{v}$ and $\theta_{13}^{v}$. A similar situation takes place in the sixth case, in which the matrices $\tilde{U}_{e}=R_{13}^{-1}\left(\theta_{13}^{e}\right)$ and $\tilde{U}_{v}=$ $R_{23}\left(\theta_{23}^{v}\right) R_{13}\left(\theta_{13}^{v}\right) R_{12}\left(\theta_{12}^{v}\right)$ imply

$$
U=R_{13}\left(\theta_{13}^{e}\right) \Psi R_{23}\left(\theta_{23}^{v}\right) R_{13}\left(\theta_{13}^{v}\right) R_{12}\left(\theta_{12}^{v}\right) Q_{0} .
$$

This parametrisation of the PMNS matrix leads to

$$
\sin ^{2} \theta_{23}=\frac{\left|U_{\mu 3}\right|^{2}}{1-\left|U_{e 3}\right|^{2}}=\frac{\sin ^{2} \theta_{23}^{v} \cos ^{2} \theta_{13}^{v}}{1-\sin ^{2} \theta_{13}},
$$

and

$$
\begin{aligned}
\cos \delta= & -\frac{1}{\sin 2 \theta_{12} \sin \theta_{13}\left|\cos \theta_{13}^{v} \sin \theta_{23}^{v}\right|\left(\cos ^{2} \theta_{13}-\cos ^{2} \theta_{13}^{v} \sin ^{2} \theta_{23}^{v}\right)^{\frac{1}{2}}} \\
& \times\left[\left(\cos ^{2} \theta_{13}-\cos ^{2} \theta_{13}^{v} \sin ^{2} \theta_{23}^{v}\right) \sin ^{2} \theta_{12}+\cos ^{2} \theta_{12} \sin ^{2} \theta_{13} \cos ^{2} \theta_{13}^{v} \sin ^{2} \theta_{23}^{v}\right. \\
& \left.-\cos ^{2} \theta_{13}\left(\cos \theta_{12}^{v} \sin \theta_{13}^{v} \sin \theta_{23}^{v}+\sin \theta_{12}^{v} \cos \theta_{23}^{v}\right)^{2}\right]
\end{aligned}
$$


It is worth noting that the sum rules presented here are exact within the schemes considered. In the following section we use these sum rules to obtain predictions for $\cos \delta$. A simplified analysis employing the best fit values of the neutrino mixing parameters $\sin ^{2} \theta_{12}, \sin ^{2} \theta_{13}$ and $\sin ^{2} \theta_{23}$ is followed by the results of the statistical analysis of the predictions performed in [23] and [20], which is based on the prospective uncertainties in the measured values of $\sin ^{2} \theta_{12}, \sin ^{2} \theta_{13}$ and $\sin ^{2} \theta_{23}$.

\section{Predictions}

We show in Table 1 the predictions for $\cos \delta$ for all the schemes considered in the present study using the current best fit values of the neutrino mixing parameters $\sin ^{2} \theta_{12}, \sin ^{2} \theta_{13}$ and $\sin ^{2} \theta_{23}$ for the neutrino mass spectrum with normal ordering (NO) taken from [10], which enter into the sum rules for $\cos \delta$ given in Eqs. (2.3), (2.6), (2.8), (2.10), (2.13) and (2.16). In the case of the BM (LC) symmetry form of $\tilde{U}_{v}$, for the current best fit values of $\sin ^{2} \theta_{12}, \sin ^{2} \theta_{13}$ and $\sin ^{2} \theta_{23}$ the corresponding sum rules give unphysical values of $\cos \delta$ (for explanation see [18, 19]).

\begin{tabular}{llcrll}
\hline Scheme & TBM & GRA & \multicolumn{1}{l}{ GRB } & \multicolumn{1}{l}{ HG } & BM (LC) \\
\hline$\theta_{12}^{e}-\left(\theta_{23}^{v}, \theta_{12}^{v}\right)$ & -0.114 & 0.289 & -0.200 & 0.476 & - \\
$\theta_{13}^{e}-\left(\theta_{23}^{v}, \theta_{12}^{v}\right)$ & 0.114 & -0.289 & 0.200 & -0.476 & - \\
$\left(\theta_{12}^{e}, \theta_{23}^{e}\right)-\left(\theta_{23}^{v}, \theta_{12}^{v}\right)$ & -0.091 & 0.275 & -0.169 & 0.445 & - \\
$\left(\theta_{13}^{e}, \theta_{23}^{e}\right)-\left(\theta_{23}^{v}, \theta_{12}^{v}\right)$ & 0.151 & -0.315 & 0.251 & -0.531 & - \\
\hline Scheme & {$[\pi / 20,-\pi / 4]$} & {$[\pi / 10,-\pi / 4]$} & {$[a,-\pi / 4]$} & {$[\pi / 20, b]$} & {$[\pi / 20, \pi / 6]$} \\
\hline$\theta_{12}^{e}-\left(\theta_{23}^{v}, \theta_{13}^{v}, \theta_{12}^{v}\right)$ & -0.222 & 0.760 & 0.911 & -0.775 & -0.562 \\
\hline Scheme & {$[\pi / 20, c]$} & {$[\pi / 20, \pi / 4]$} & {$[\pi / 10, \pi / 4]$} & {$[a, \pi / 4]$} & {$[\pi / 20, d]$} \\
\hline$\theta_{13}^{e}-\left(\theta_{23}^{v}, \theta_{13}^{v}, \theta_{12}^{v}\right)$ & -0.866 & 0.222 & -0.760 & -0.911 & -0.791 \\
\hline
\end{tabular}

Table 1: The predicted values of $\cos \delta$ using the current best fit values of $\sin ^{2} \theta_{12}, \sin ^{2} \theta_{13}$ and $\sin ^{2} \theta_{23}$ for the NO neutrino mass spectrum taken from [10]. We have defined $a=\sin ^{-1}(1 / 3), b=\sin ^{-1}(1 / \sqrt{2+r})$, $c=\sin ^{-1}(1 / \sqrt{3})$ and $d=\sin ^{-1}(\sqrt{3-r} / 2)$. For the last two schemes we give in square brackets the values of $\left[\theta_{13}^{v}, \theta_{12}^{v}\right]$. TBM, GRA, GRB, HG and BM (LC) refer, in particular, to the different fixed values of $\theta_{12}^{v}=c$, $b, d, \pi / 6$ and $\pi / 4$, respectively. See text for further details.

In Fig. 1 we present results of a statistical analysis for the $\left(\theta_{12}^{e}, \theta_{23}^{e}\right)-\left(\theta_{23}^{v}, \theta_{12}^{v}\right)$ and $\left(\theta_{13}^{e}, \theta_{23}^{e}\right)-$ $\left(\theta_{23}^{v}, \theta_{12}^{v}\right)$ schemes, in which the best fit values of all the three neutrino mixing angles can be reproduced, provided $\tilde{U}_{v}$ has the TBM, GRA, GRB or HG symmetry form. This analysis is performed using the procedure described in [23] (see also [24]), which allows one to get the dependence of the $\chi^{2}$ function, and hence the likelihood function $L$, defined as

$$
L(\cos \delta) \propto \exp \left(-\frac{\chi^{2}(\cos \delta)}{2}\right)
$$

on $\cos \delta$. The likelihood function represents the most probable values of $\cos \delta$ for each of the symmetry forms considered. The maxima of $L$ for the different symmetry forms of $\tilde{U}_{v}$ correspond 

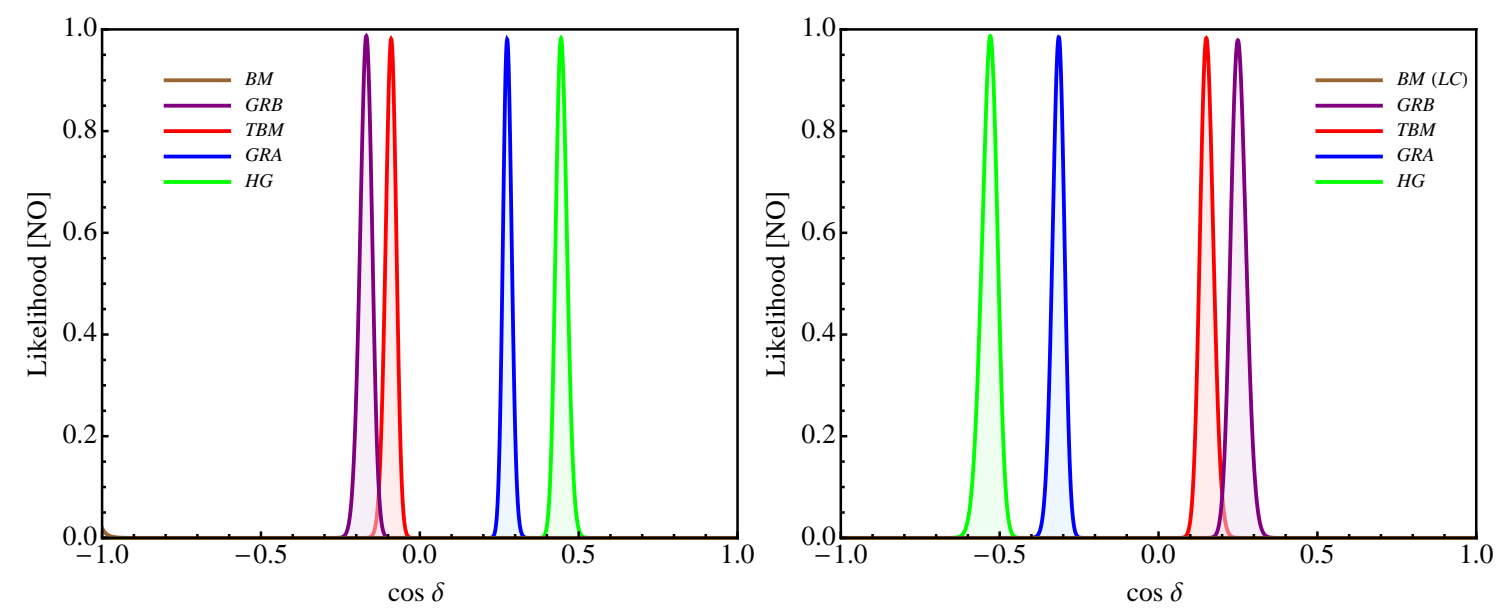

Figure 1: The likelihood function versus $\cos \delta$ for the NO neutrino mass spectrum after marginalising over $\sin ^{2} \theta_{13}$ and $\sin ^{2} \theta_{23}$ for the TBM, BM (LC), GRA, GRB and HG symmetry forms of the matrix $\tilde{U}_{v}$ in the $\left(\theta_{12}^{e}, \theta_{23}^{e}\right)-\left(\theta_{23}^{v}, \theta_{12}^{v}\right)$ scheme (left panel) and the $\left(\theta_{13}^{e}, \theta_{23}^{e}\right)-\left(\theta_{23}^{v}, \theta_{12}^{v}\right)$ scheme (right panel). The results shown are obtained using the prospective $1 \sigma$ uncertainties on $\sin ^{2} \theta_{12}, \sin ^{2} \theta_{13}, \sin ^{2} \theta_{23}$ and the Gaussian approximation for the likelihood function. See text for further details.

to the values of $\cos \delta$ given in Table 1 . The results shown are obtained by marginalising over $\sin ^{2} \theta_{13}$ and $\sin ^{2} \theta_{23}$ for a fixed value of $\delta$ (for details of the statistical analysis see [23]). The $n \sigma$ confidence level region corresponds to the interval of values of $\cos \delta$ for which $L(\cos \delta) \geq L\left(\chi^{2}=\right.$ $\left.\chi_{\min }^{2}\right) \cdot L\left(\chi^{2}=n^{2}\right)$. Here $\chi_{\min }^{2}$ is the value of $\chi^{2}$ in the minimum.

We present $L$ versus $\cos \delta$ within the Gaussian approximation, i.e., constructing $\chi^{2}$ as a sum: $\chi^{2}=\sum_{i=1}^{3}\left(x_{i}-\bar{x}_{i}\right)^{2} / \sigma_{x_{i}}^{2}$, where $x_{i}=\left(\sin ^{2} \theta_{12}, \sin ^{2} \theta_{13}, \sin ^{2} \theta_{23}\right)$. We employ the current best fit values $\left(\bar{x}_{i}\right)$ of $\sin ^{2} \theta_{12}, \sin ^{2} \theta_{13}, \sin ^{2} \theta_{23}$ for the NO spectrum obtained in [10] and the prospective $1 \sigma$ uncertainties $\left(\sigma_{x_{i}}\right)$ in the measurement of these mixing parameters. More specifically, we use as $1 \sigma$ uncertainties a) $0.7 \%$ for $\sin ^{2} \theta_{12}$, which is the prospective sensitivity of the JUNO experiment [25], b) $5 \%$ for $\sin ^{2} \theta_{23}$, obtained from the prospective uncertainty of $2 \%$ [3] on $\sin ^{2} 2 \theta_{23}$ expected to be reached in the NOvA and T2K experiments, and c) $3 \%$ for $\sin ^{2} \theta_{13}$, deduced from the error of $3 \%$ on $\sin ^{2} 2 \theta_{13}$ planned to be reached in the Daya Bay experiment $[3,26]$.

As can be observed from Fig. 1, a rather precise measurement of $\cos \delta$ would allow one to distinguish between the different symmetry forms of $\tilde{U}_{v}$ considered by us. The BM case is very sensitive to the best fit values of $\sin ^{2} \theta_{12}$ and $\sin ^{2} \theta_{23}$ and is disfavoured at more than $2 \sigma$ for the current best fit values. This case might turn out to be compatible with the data for larger (smaller) measured values of $\sin ^{2} \theta_{12}\left(\sin ^{2} \theta_{23}\right)$ as demonstrated in [23]. Comparing the left and right panels of Fig. 1, we note first that for a given symmetry form, $\cos \delta$ is predicted to have opposite signs in the two schemes. In the scheme $\left(\theta_{13}^{e}, \theta_{23}^{e}\right)-\left(\theta_{23}^{v}, \theta_{12}^{v}\right)$ (right panel) one has $\cos \delta>0$ in the TBM and GRB cases, while $\cos \delta<0$ in the cases of the GRA and HG symmetry forms. It is also important to note that due to the fact that the best fit value of $\sin ^{2} \theta_{23}<0.5$, the predictions for $\cos \delta$ for each symmetry form, obtained in the two set-ups, differ not only by sign but also in absolute value. Thus, a precise measurement of $\cos \delta$ would allow one to distinguish not only between the symmetry forms of $\tilde{U}_{v}$, but also could provide an indication about the structure of the matrix $\tilde{U}_{e}$. 


\section{Conclusions}

In conclusion, we presented the sum rules for the cosine of the Dirac phase $\delta$ in the PMNS mixing matrix, which is assumed to have a particular form dictated by symmetry arguments. These sum rules are exact within the approach employed. The results obtained in [19, 20, 23] and summarised in this note show that the measurement of the Dirac phase $\delta$ in the PMNS mixing matrix along with improvement of the precision on the mixing angles $\theta_{12}, \theta_{13}$ and $\theta_{23}$ can provide unique information as regards the possible existence of (discrete) symmetry in the lepton sector. These measurements could also provide an indication about the charged lepton mass matrix.

\section{Acknowledgments}

A.T. thanks the organisers of the European Physical Society Conference on High Energy Physics 2015 for the opportunity to present this work. It was supported in part by the European Union FP7 ITN INVISIBLES (Marie Curie Actions, PITN-GA-2011-289442-INVISIBLES), by the INFN program on Theoretical Astroparticle Physics (TASP), by the research grant 2012CPPYP7 (Theoretical Astroparticle Physics) under the program PRIN 2012 funded by the Italian Ministry of Education, University and Research (MIUR) and by the World Premier International Research Center Initiative (WPI Initiative, MEXT), Japan (S.T.P.).

\section{References}

[1] K. Nakamura and S. T. Petcov, in K. A. Olive et al. (Particle Data Group), Chin. Phys. C 38 (2014) 090001.

[2] S. K. Agarwalla et al. [LAGUNA-LBNO Collaboration], JHEP 1405 (2014) 094; C. Adams et al. [LBNE Collaboration], arXiv:1307.7335 [hep-ex].

[3] A. de Gouvea et al. [Intensity Frontier Neutrino Working Group Collaboration], arXiv:1310.4340 [hep-ex].

[4] C. Giunti and M. Tanimoto, Phys. Rev. D 66 (2002) 113006; see also: C. Giunti and M. Tanimoto, Phys. Rev. D 66 (2002) 053013.

[5] Z. z. Xing, Phys. Lett. B 533 (2002) 85.

[6] P. H. Frampton, S. T. Petcov and W. Rodejohann, Nucl. Phys. B 687 (2004) 31.

[7] S. T. Petcov and W. Rodejohann, Phys. Rev. D 71 (2005) 073002.

[8] A. Romanino, Phys. Rev. D 70 (2004) 013003.

[9] S. M. Bilenky, J. Hosek and S. T. Petcov, Phys. Lett. B 94 (1980) 495.

[10] F. Capozzi et al., Phys. Rev. D 89 (2014) 093018.

[11] M. C. Gonzalez-Garcia, M. Maltoni and T. Schwetz, JHEP 1411 (2014) 052.

[12] P. F. Harrison, D. H. Perkins and W. G. Scott, Phys. Lett. B 530 (2002) 167; P. F. Harrison and W. G. Scott, Phys. Lett. B 535 (2002) 163; X. G. He and A. Zee, Phys. Lett. B 560 (2003) 87; see also: L. Wolfenstein, Phys. Rev. D 18 (1978) 958.

[13] S. T. Petcov, Phys. Lett. B 110 (1982) 245. 
[14] F. Vissani, hep-ph/9708483; V. D. Barger, S. Pakvasa, T. J. Weiler and K. Whisnant, Phys. Lett. B 437 (1998) 107; A. J. Baltz, A. S. Goldhaber and M. Goldhaber, Phys. Rev. Lett. 81 (1998) 5730.

[15] A. Datta, F. S. Ling and P. Ramond, Nucl. Phys. B 671 (2003) 383; Y. Kajiyama, M. Raidal and A. Strumia, Phys. Rev. D 76 (2007) 117301; L. L. Everett and A. J. Stuart, Phys. Rev. D 79 (2009) 085005 .

[16] W. Rodejohann, Phys. Lett. B 671 (2009) 267; A. Adulpravitchai, A. Blum and W. Rodejohann, New J. Phys. 11 (2009) 063026.

[17] C. H. Albright, A. Dueck and W. Rodejohann, Eur. Phys. J. C 70 (2010) 1099; J. E. Kim and M. S. Seo, JHEP 1102 (2011) 097.

[18] D. Marzocca, S. T. Petcov, A. Romanino and M. C. Sevilla, JHEP 1305 (2013) 073.

[19] S. T. Petcov, Nucl. Phys. B 892 (2015) 400.

[20] I. Girardi, S. T. Petcov and A. V. Titov, Eur. Phys. J. C 75 (2015) 7, 345.

[21] I. Girardi, S. T. Petcov, A. J. Stuart and A. V. Titov, arXiv:1509.02502 [hep-ph].

[22] F. Bazzocchi, arXiv:1108.2497 [hep-ph]; W. Rodejohann and H. Zhang, Phys. Lett. B 732 (2014) 174; R. d. A. Toorop, F. Feruglio and C. Hagedorn, Phys. Lett. B 703 (2011) 447; G. J. Ding, Nucl. Phys. B 862 (2012) 1; S. F. King, C. Luhn and A. J. Stuart, Nucl. Phys. B 867 (2013) 203.

[23] I. Girardi, S. T. Petcov and A. V. Titov, Nucl. Phys. B 894 (2015) 733.

[24] I. Girardi, S. T. Petcov and A. V. Titov, Int. J. Mod. Phys. A 30 (2015) 13, 1530035; I. Girardi, S. T. Petcov and A. V. Titov, J. Phys. Conf. Ser. 631 (2015) 1, 012051.

[25] Y. Wang, PoS (Neutel 2013)030.

[26] C. Zhang [Daya Bay Collaboration], AIP Conf. Proc. 1666 (2015) 080003. 